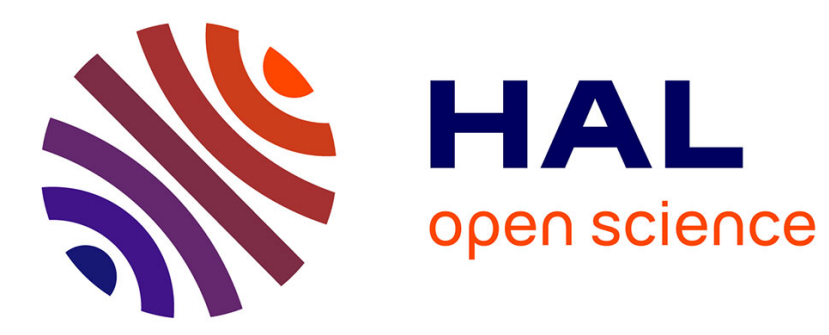

\title{
Estimation of vehicle lateral tire-road forces: a comparison between extended and unscented Kalman filtering
}

\author{
Moustapha Doumiati, Alessandro Victorino, Ali Charara, Daniel Lechner
}

\section{- To cite this version:}

Moustapha Doumiati, Alessandro Victorino, Ali Charara, Daniel Lechner. Estimation of vehicle lateral tire-road forces: a comparison between extended and unscented Kalman filtering. ECC 09, Aug 2009, Hungary. pp.6. hal-00426299

\section{HAL Id: hal-00426299 \\ https://hal.science/hal-00426299}

Submitted on 24 Oct 2009

HAL is a multi-disciplinary open access archive for the deposit and dissemination of scientific research documents, whether they are published or not. The documents may come from teaching and research institutions in France or abroad, or from public or private research centers.
L'archive ouverte pluridisciplinaire HAL, est destinée au dépôt et à la diffusion de documents scientifiques de niveau recherche, publiés ou non, émanant des établissements d'enseignement et de recherche français ou étrangers, des laboratoires publics ou privés. 


\title{
Estimation of vehicle lateral tire-road forces: a comparison between extended and unscented Kalman filtering
}

\author{
Moustapha Doumiati, Alessandro Victorino, Ali Charara and Daniel Lechner
}

\begin{abstract}
Extensive research has shown that most of road accidents occur as a result of driver errors. A close examination of accident data reveals that losing the vehicle control is responsible for a huge proportion of car accidents. Preventing such kind of accidents using vehicle control systems, requires certain input data concerning vehicle dynamic parameters and vehicle road interaction. Unfortunately, some parameters like tire-road forces and sideslip angle, which have a major impact on vehicle dynamics, are difficult to measure in a car. Therefore, this data must be estimated. Due to the system nonlinearities and unmodeled dynamics, two observers derived from extended and unscented Kalman filtering techniques are proposed and compared. The estimation process method is based on the dynamic response of a vehicle instrumented with cheap, easily-available standard sensors. Performances are tested and compared to real experimental data acquired using the INRETS-MA Laboratory car. Experimental results demonstrate the ability of this approach to provide accurate estimations, and show its practical potential as a low-cost solution for calculating lateral-tire forces and sideslip angle.
\end{abstract}

\section{INTRODUCTION}

Vehicle control algorithms such as Electronic Stability Control (ESC) systems have made great strides towards improving the handling and safety of vehicles. In fact, experts estimate that the ESC prevents $27 \%$ of loss of control accidents by intervening when emergency situations are detected [1]. While ESC is undoubtedly a life-saving technology, it is limited by the available vehicle state information.

ESC systems currently available on production cars rely on avaible inexpensive measurements (such as longitudinal velocity, accelerations and yaw rate), tire model, and sideslip rate, not sideslip angle. Calculating sideslip angle from sideslip rate integration is prone to uncertainty and errors from sensor biases. Furthermore, other essential parameters like tire-road forces are difficult to measure because of technical, physical and economic reasons. Therefore, these important data must be observed or estimated. If control systems could characterize lateral tire forces characteristics, namely lateral forces, sideslip angle and tire-road friction coefficient, these systems could greatly enhance vehicle handling and increase passenger safety.

As the motion of a vehicle is governed by the forces generated between the tires and the road, knowledge of the tire forces is crucial to predicting vehicle motion. The lateral forces necessary for a vehicle to hold a curve arise as a result of tire deformation. As shown in figure 1, the relationship between the lateral force and the slip angle is initially linear with a constant slope of $C_{\alpha}$, referred to as the cornering stiffness. When operating in the linear region, a vehicle responds predictably to the driver's inputs. When a vehicle undergoes high accelerations, or when road friction changes, the vehicle dynamic becomes nonlinear and the force begins to saturate. Consequently, the tire enters the nonlinear operating region and the vehicle approaches its handling limits and its response becomes less predictable.

M. Doumiati, A Victorino and A Charara are with Heudiasyc Laboratory, UMR CNRS 6599, Université de Technologie de Compiègne, 60205 Compiègne, France mdoumiatahds.utc. fr, acorreavehds.utc. fr and achararaehds.utc. fr D. Lechner is with Inrets-MA Laboratory, Departement of Accident Mechanism Analysis, Chemin de la Croix Blanche, 13300 Salon de Provence, France daniel. lechnerdinrets.fr
Lateral vehicle dynamic estimation has been widely discussed in the literature. Several studies have been conducted regarding the estimation of tire-road forces and sideslip angle [2]-[9]. For example, in [2] and [3], the authors estimate the vehicle dynamic state for a four-wheel vehicle model. Consequently, tire forces are calculated based on the estimated states and using tire models. In [4], Ray estimates the vehicle dynamic states and lateral tire forces per axle for a nine DOF vehicle model. The author uses measures of the applied torques as inputs to his model. We note that the torque is difficult to get in practice; it requires expensive sensors. More recently, in [5] and [6], authors propose observers to estimate lateral forces per axle without using torque measures. In [7], the authors propose an estimation process based on a three DOF vehicle model, as a tire force estimator. In [8] and [9], sideslip angle estimation is discussed in details.

In [5], [6], [7], lateral forces are modelled with a derivative equal to random noise. The authors in [7], remark that such modeling leads to a noticeable inaccuracy when estimating individual lateral tire forces, but not in axle lateral forces. This phenomenon is due to the non-representation of the lateral load transfer when modeling [7].

The main goal of this study is to develop an estimation method that uses a simple vehicle-road model and a certain number of valid measurements in order to estimate accuracy and in real-time the lateral force at each individual tireroad contact point. We suppose a prior knowledge of road conditions. This study presents two particularities:

- the estimation process does not use the measurement of wheel torques,

- As described in section II, the estimation process uses accurate estimated normal tire forces, while other approaches found in the literature assume constant vertical forces.

The observation system is highly nonlinear and presents unmodeled dynamics. For this reason, two observers based on EKF (Extended Kalman Filter) and UKF (Unscented Kalman Filter) are proposed. The EKF is probably the most used estimator for nonlinear systems, however the UKF has shown the ability to be a superior alternative especially when system presents strong nonlinearities. This study compares and discusses this two filtering techniques in our estimation approach.

In order to show the effectiveness of the estimation method, some validation tests were carried out on an instrumented vehicle in realistic driving situations.

The remainder of the paper is organized as follows. In section 2 we describe the estimation process. Section 3 presents the vehicle/road model. Section 4 describes the observer and presents the observability analysis. In section 5 the observers results are discussed and compared to real experimental data, and then in the final section we make some concluding remarks regarding our study and future perspectives.

\section{ESTIMATION PROCESS DESCRIPTION}

The estimation process is shown in its entirety by the block diagram in figure 2, where $a_{x}$ and $a_{y m}$ are respectively the longitudinal and lateral accelerations, $\dot{\psi}$ is the yaw rate, $\Delta_{i j}$ ( $i$ represents front(1) or rear(2) and $j$ represents left(1) 


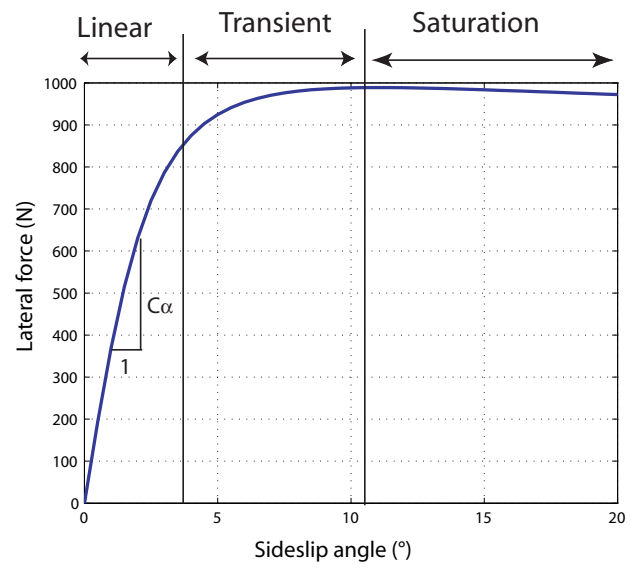

Fig. 1. Generic tire curve

or right (2)) is the suspension deflection, $w_{i j}$ is the wheel velocity, $F_{z i j}$ and $F_{y i j}$ are respectively the normal and lateral tire/road forces, $\beta$ is the sideslip angle at the centre of gravity (cog). The estimation process consists of two blocks and its role is to estimate normal and lateral forces at each tire/road level and then to evaluate the lateral friction coefficient. The following measurements are needed:

- yaw rate, longitudinal and lateral accelerations measured by an inertial sensor,

- suspension deflections using suspension deflections sensors,

- steering angle measured by an optical sensor,

- rotational velocity for each tire given by ABS.

The first block aims to provide the vehicle's weight, normal tire forces and the corrected lateral acceleration $a_{y}$ (by canceling the gravitational acceleration that distorts the accelerometer signal). It contains observers based on vehicle's roll dynamics and model that couples longitudinal and lateral accelerations. The first block was the subject of our previous studies [10], [11]. This article focuses only on the second block, whose main role is to estimate lateral tire forces and sideslip angle. The second block makes use of the estimations provided by the first block. One particularity of this estimation process is the use of blocks in series. By using cascaded observers, the observability problems entailed by an inappropriate use of the complete modeling equations are avoided enabling the estimation process to be carried out in a simple and practical way.

\section{VEHICLE-ROAD MODEL}

\section{A. Four-wheel vehicle model}

The Four-Wheel Vehicle model (FWVM) was chosen for this study because it is simple and corresponds sufficiently to our objectives. The FWVM is widely used to describe transversal vehicle dynamic behavior [3], [4], [6]. In this study, we adopt the following simplified assumptions:

- rear longitudinal forces are neglected relative to the front longitudinal forces (Assuming a front-driven vehicle). We suppose that $F_{x 1}=F_{x 11}+F_{x 12}$,

- front steering angles are equal $\left(\delta_{11}=\delta_{12}=\delta\right)$ and rear steering angles are approximately null $\left(\delta_{21}=\delta_{22}=0\right)$.

Figure 3 shows the FWVM model, where $\dot{\psi}$ is the yaw rate, $\beta$ is the center of gravity sideslip angle, it is the angle between the vehicle heading and the direction of its velocity, $V_{g}$ the center of gravity velocity, and $L_{1}$ and $L_{2}$ the distance from the vehicle center of gravity to the front and rear axles

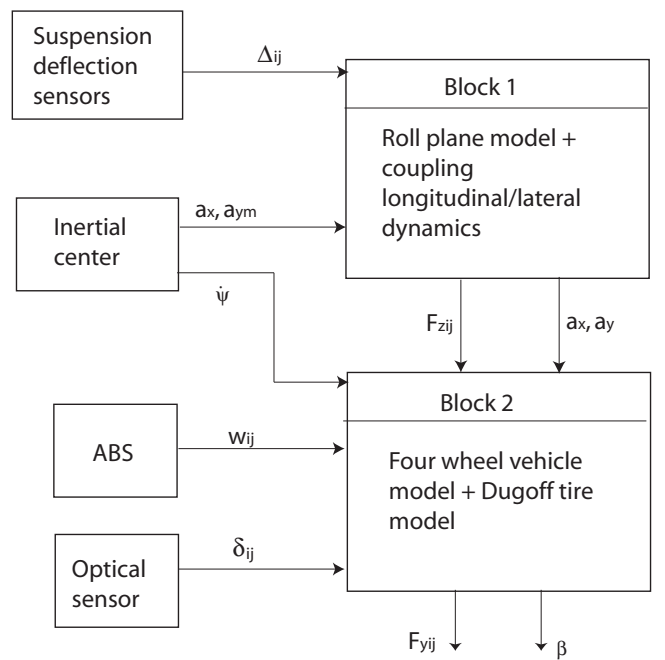

Fig. 2. Process estimation diagram

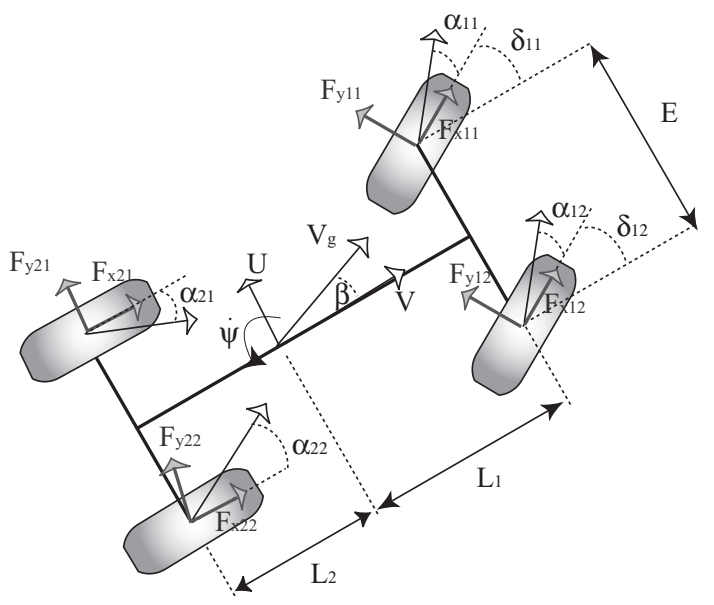

Fig. 3. Four-wheel vehicle model.

respectively. $F_{x, y, i, j}$ are the longitudinal and lateral tireroad forces, $\delta_{1,2}$ are the front left and right steering angles respectively, and $E$ is the vehicle track (lateral distance from wheel to wheel).

The simplified FWVM is derived from [12] and it is formulated as the following dynamic relationship:

$$
\begin{aligned}
& \dot{V}_{g}=\frac{1}{m}\left[\begin{array}{l}
F_{x 1} \cos (\beta-\delta)+F_{y 11} \sin (\beta-\delta)+ \\
F_{y 12} \sin (\beta-\delta)+\left(F_{y 21}+F_{y 22}\right) \sin (\beta)
\end{array}\right], \\
& \ddot{\psi}=\frac{1}{I_{z}}\left[\begin{array}{l}
L_{1}\left[F_{y 11} \cos \delta+F_{y 12} \cos \delta+F_{x 1} \sin \delta\right]- \\
L_{2}\left[F_{y 21}+F_{y 22}\right]+ \\
\frac{E}{2}\left[F_{y 11} \sin \delta-F_{y 12} \sin (\delta)\right]
\end{array}\right], \\
& \dot{\beta}=\frac{1}{m V_{g}}\left[\begin{array}{l}
-F_{x 1} \sin (\beta-\delta)+F_{y 11} \cos (\beta-\delta)+ \\
F_{y 12} \cos (\beta-\delta)+\left(F_{y 21}+F_{y 22}\right) \cos (\beta)
\end{array}\right]-\dot{\psi}, \\
& a_{y}=\frac{1}{m}\left[F_{y 11} \cos \delta+F_{y 12} \cos \delta+\left(F_{y 21}+F_{y 22}\right)+F_{x 1} \sin \delta\right], \\
& a_{x}=\frac{1}{m}\left[-F_{y 11} \sin \delta-F_{y 12} \sin \delta+F_{x 1} \cos \delta\right] .
\end{aligned}
$$

where $m$ is the vehicle mass, $I_{z}$ is the yaw moment of inertia and $\alpha_{i j}$ are the front and the rear sideslip angles (tire sideslip 
angle is the angle between the tire direction and its velocity). The vehicle velocity $V_{g}$, the steer angle $\delta$, the yaw rate $\psi$ and the vehicle body slip angle $\beta$ are then used as a basis for the calculation of the tyre slip angles $\alpha_{i j}$, where:

$$
\begin{aligned}
& \alpha_{11}=\delta-\arctan \left[\frac{V_{g} \beta+L_{1} \dot{\psi}}{V_{g}-E \dot{\psi} / 2}\right], \\
& \alpha_{12}=\delta-\arctan \left[\frac{V_{g} \beta+L_{1} \dot{\psi}}{V_{g}+E \dot{\psi} / 2}\right], \\
& \alpha_{21}=-\arctan \left[\frac{V_{g} \beta-L_{2} \dot{\psi}}{V_{g}-E \dot{\psi} / 2}\right], \\
& \alpha_{22}=-\arctan \left[\frac{V_{g} \beta-L_{2} \dot{\psi}}{V_{g}+E \dot{\psi} / 2}\right] .
\end{aligned}
$$

\section{B. Lateral tire-force model}

The model of tire-road contact forces is complex because a wide variety of parameters including environmental factors and pneumatic properties (load, tire pressure, etc.) impact the tire-road contact interface. Many different tire models are to be found in the literature, based on the physical nature of the tire and/or on empirical formulations deriving from experimental data, such as the Pacejka, Dugoff and Burckhardt models [12], [13]. Dugoff's model was selected for this study because of the small number of parameters that are sufficient to evaluate the tire-road forces. The nonlinear lateral tire forces are given by:

$$
F_{y i j}=-C_{\alpha i} \tan \alpha_{i j} \cdot f(\lambda)
$$

where $C_{\alpha i}$ is the lateral stiffness, $\alpha_{i j}$ is the slip angle and $f(\lambda)$ is given by:

$$
\begin{gathered}
f(\lambda)= \begin{cases}(2-\lambda) \lambda, & \text { if } \lambda<1 \\
1, & \text { if } \lambda \geq 1\end{cases} \\
\lambda=\frac{\mu F_{z i j}}{2 C_{\alpha_{i}} \mid{\text { tan } \alpha_{i j} \mid}}
\end{gathered}
$$

In the above formulation, $\mu$ is the coefficient of friction and $F_{z i j}$ is the normal load on the tire. This simplified tire model assumes no longitudinal forces, a uniform pressure distribution, a rigid tire carcass, and a constant coefficient of friction of sliding rubber [14].

\section{Relaxation model}

When vehicle sideslip angle changes, a lateral tire force is created with a time lag. This transient behavior of tires can be formulated using a relaxation length $\sigma$. The relaxation length is the distance covered by the tire while the tire force is kicking in. Using the relaxation model presented in [15], lateral forces can be written as:

$$
\dot{F}_{y i j}=\frac{V_{g}}{\sigma_{i}}\left(-F_{y i j}+\overline{F_{y i j}}\right),
$$

where $\overline{F y_{i j}}$ is calculated from a Dugoff's reference tire-force model, $V_{g}$ is the vehicle velocity and $\sigma_{i}$ is the relaxation length.

\section{OBSERVER DESIGN}

This section presents a description of the observer dedicated to lateral tire forces and sideslip angle. The nonlinear stochastic state-space representation of the system described in the section above is given as:

$$
\left\{\begin{array}{l}
\dot{X}(t)=f(X(t), U(t))+b_{m}(t) \\
Y(t)=h(X(t), U(t))+b_{s}(t)
\end{array}\right.
$$

The input vector $U$ comprises the steering angle and the normal forces considered estimated by the first block (see section 2):

$U=\left[\delta, F_{z 11}, F_{z 12}, F_{z 21}, F_{z 22}\right]=\left[u_{1}, u_{2}, u_{3}, u_{4}, u_{5}\right]$.

The measure vector $\mathrm{Y}(\mathrm{t})$ comprises yaw rate, vehicle velocity (approximated by the mean of the rear wheel velocities calculated from wheel encoders information), longitudinal and lateral accelerations:

$$
Y=\left[\dot{\psi}, V_{g}, a_{x}, a_{y}\right]=\left[y_{1}, y_{2}, y_{3}, y_{4}\right] .
$$

The state vector comprises yaw rate, vehicle velocity, sideslip angle at the $\operatorname{cog}$, lateral forces and the sum of the front longitudinal tire forces:

$$
\begin{aligned}
X & =\left[\dot{\psi}, V_{g}, \beta, F_{y 11}, F_{y 12}, F_{y 21}, F_{y 22}, F_{x 1}\right] \\
& =\left[x_{1}, x_{2}, x_{3}, x_{4}, x_{5}, x_{6}, x_{7}, x_{8}\right] .
\end{aligned}
$$

The process and measurement noise vectors, respectively $b_{m}(t)$ and $b_{s}(t)$, are assumed to be white, zero mean and uncorrelated.

Consequently, the evolution equations are:

$$
\begin{aligned}
\dot{X} & =f(X, U)=\left[\dot{x_{1}}, \dot{x_{2}}, \dot{x_{3}}, \dot{x_{4}}, \dot{x_{5}}, \dot{x_{6}}, \dot{x_{7}}, \dot{x_{8}}\right] \\
\dot{x_{1}} & =\frac{1}{I_{z}}\left[\begin{array}{l}
L_{1}\left[x_{4} \cos u_{1}+x_{5} \cos u_{1}+x_{8} \sin u_{1}\right]- \\
L_{2}\left[x_{6}+x_{7}\right]+ \\
\frac{E}{2}\left[x_{4} \sin u_{1}-x_{5} \sin u_{1}\right]
\end{array}\right], \\
\dot{x_{2}} & =\frac{1}{m}\left[\begin{array}{l}
x_{8} \cos \left(x_{3}-u_{1}\right)+x_{4} \sin \left(x_{3}-u_{1}\right)+ \\
x_{5} \sin \left(x_{3}-u_{1}\right)+\left(x_{6}+x_{7}\right) \sin \left(x_{3}\right)
\end{array}\right], \\
\dot{x_{3}} & =\frac{1}{m V_{g}}\left[\begin{array}{l}
-x_{8} \sin \left(x_{3}-u_{1}\right)+x_{4} \cos \left(x_{3}-u_{1}\right)+ \\
x_{5} \cos \left(x_{3}-u_{1}\right)+\left(x_{6}+x_{7}\right) \cos x_{3}
\end{array}\right]-x_{1}, \\
\dot{x_{4}} & =\frac{x_{2}}{\sigma_{1}}\left(-x_{4}+\overline{F_{y 11}}\left(\alpha_{11}, u_{2}\right)\right), \\
\dot{x_{5}} & =\frac{x_{2}}{\sigma_{1}}\left(-x_{5}+\overline{F_{y 12}}\left(\alpha_{12}, u_{3}\right)\right), \\
\dot{x_{6}} & =\frac{x_{2}}{\sigma_{2}}\left(-x_{6}+\overline{F_{y 21}}\left(\alpha_{21}, u_{4}\right)\right), \\
\dot{x_{7}} & =\frac{x_{2}}{\sigma_{2}}\left(-x_{7}+\overline{F_{y 22}}\left(\alpha_{22}, u_{5}\right)\right), \\
\dot{x_{8}} & =0 .
\end{aligned}
$$

The observation equations are:

$$
\begin{aligned}
& y_{1}=x_{1}, \\
& y_{2}=x_{2} \\
& y_{3}=\frac{1}{m}\left[-x_{4} \sin u_{1}-x_{5} \sin u_{1}+x_{8} \cos u_{1}\right] \\
& y_{4}=\frac{1}{m}\left[x_{4} \cos u_{1}+x_{5} \cos u_{1}+\left(x_{6}+x_{7}\right)+x_{6} \sin u_{1}\right] .
\end{aligned}
$$

The state vector $X(t)$ will be estimated by applying the extended and unscented Kalman filter techniques: observers $O_{E K F}$ and $O_{U K F}$ ) respectively (see section IV-B).

\section{A. Observability}

Observability is a measure of how well the internal states of a system can be inferred from knowledge of its inputs and external outputs. This property is often presented as a rank condition on the observability matrix. Using the nonlinear state space formulation of the system represented in (6), the 

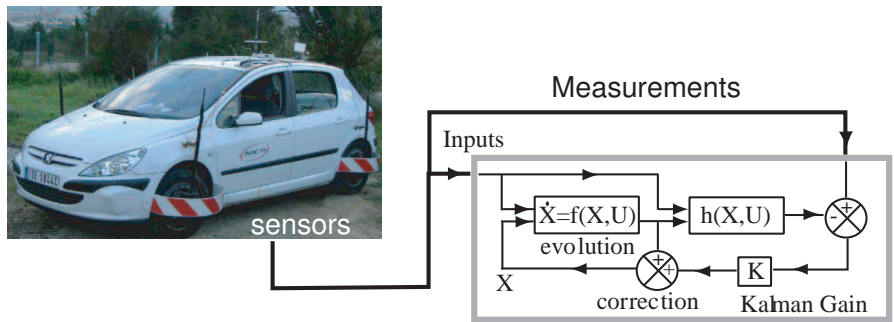

observer

Fig. 4. Process estimation diagram

observability definition is local and uses the Lie derivative [16]. An observability analysis of this system was undertaken in [17]. It has been shown that the system is observable except when:

- steering angles are null,

- vehicle is at rest $\left(V_{g}=0\right)$.

For these situations, we assume that lateral forces and sideslip angle are null, which approximately corresponds to the real cases.

\section{B. Estimation method}

The aim of an observer or a virtual sensor is to estimate a particular unmeasurable variable from available measurements and a system model in a closed loop observation scheme, as illustrated in figure 4. A simple example of an open loop observer is the model given by relations (1). Because of the system-model mismatch (unmodelled dynamics, parameter variations,...) and the presence of unknown and unmeasurable disturbances, the calculation obtained from the open loop observer would deviate from the actual values over time. In order to reduce the estimation error, at least some of the measured outputs are compared to the same variables estimated by the observer. The difference is fed back into the observer after being multiplied by a gain matrix $K$, and so we have a closed loop observer (see figure 4). The observer was implemented in a first-order Euler approximation discrete form. At each iteration, the state vector is first calculated according to the evolution equation and then corrected online with the measurement errors (innovation) and filter gain $K$ in a recursive prediction-correction mechanism. The gain is calculated using the Kalman filter method which is a set of mathematical equations and is widely represented in [18], [19].

First, the $O_{E K F}$ has been developped in order to estimate the state vector $X(t)$ (see section IV). However, some system properties and EKF drawbacks encountered during this study, especially:

- the high nonlineaties of the model,

- the calculation complexity of the Jacobian matrices which causes implementation difficulties,

lead us to develop the $O_{U K F}$. The UKF is introduced to improve the EKF especially for strong nonlinear systems. For these systems, the first order linearization of the EKF algorithm using Jacobian matrices is not enough, and the errors linearization are too important. The UKF acts directly on the nonlinear model and approximates the states by using a set of sigma points, avoiding the linearization made by the EKF [20], [21]. The UKF is a powerful nonlinear estimation technique and has been shown to be a superior alternative to the EKF in many robotic applications.
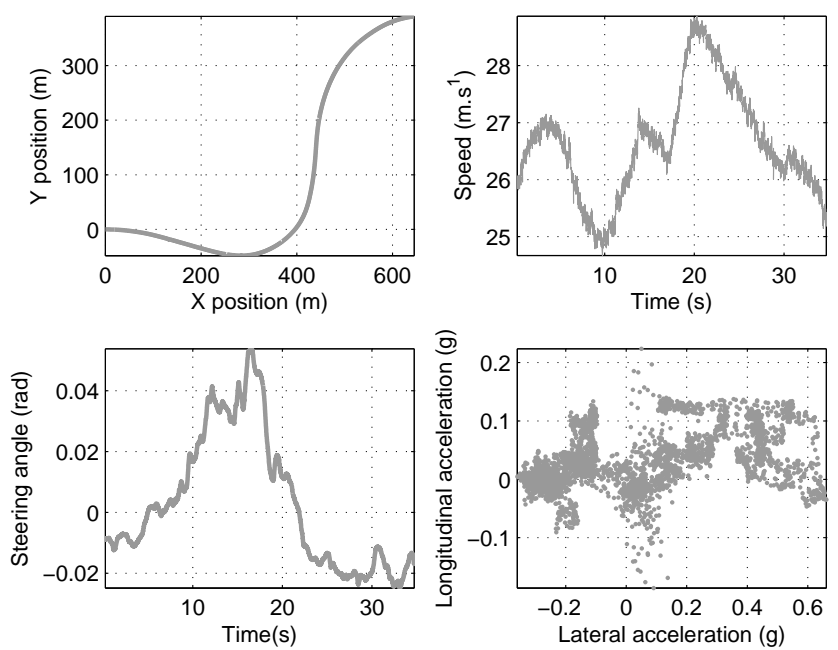

Fig. 5. Experimental test: vehicle trajectory, speed, steering angle and acceleration diagrams

\section{EXPERIMENTAL RESULTS}

\section{A. Experimental car}

The experimental vehicle shown in figure 4 is the INRETS-MA (Institut National de la Recherche sur les Transports et leur Sécurité - Département Mécanismes d'Accidents) Laboratory's test vehicle. It is a Peugeot 307 equipped with a number of sensors including accelerometers, gyrometers, steering angle sensors, linear relative suspension sensors, correvit and dynamometric hubs. Among these sensors, the correvit (a non-contact optical sensor) gives measurements of rear sideslip angle and vehicle velocity, while the dynamometric hubs are wheelforce transducers that measure in real time the forces and moments acting at the wheel center. We note that the correvit and the wheel-force transducer are very expensive sensors (correvit: $15 \mathrm{~K} €$ and dynamometric hub: $100 \mathrm{~K} €$ ). The sampling frequency of the different sensors is $100 \mathrm{~Hz}$.

The estimation process algorithm is a computer program written in $\mathrm{C}++$. It is integrated into the laboratory car as a DLL (Dynamic Link Library) that functions according to the software acquisition system.

\section{B. Test conditions}

Test data from nominal as well as adverse driving conditions were used to assess the performance of the observer presented in section IV, in realistic driving situations. We report a right-left-right bend combination maneuver (one of a number of experimental tests that we carried out) where the dynamic contributions play an important role. Figure 5 presents the Peugeot's trajectory (on a dry road), its speed, steering angle and "g-g" acceleration diagram during the course of the test. The acceleration diagram, that determines the maneuvering area utilized by the driver/vehicle, shows that large lateral accelerations were obtained (absolute value up to $0.6 g$ ). This means that the experimental vehicle was put in a critical driving situation.

\section{Validation of observers}

The observer results are presented in two forms: as tables of normalized errors, and as figures comparing the measurements and the estimations. The normalized error for 

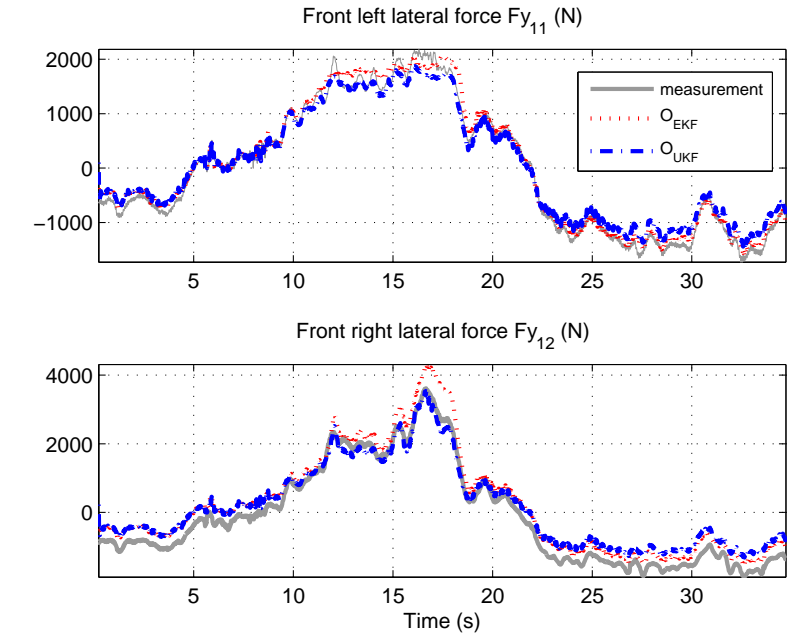

Fig. 6. Estimation of front lateral tire forces.

an estimation $z$ is defined in [8] and [9] as:

$$
\epsilon_{z}=100 \times \frac{\left\|z_{\text {obs }}-z_{\text {measured }}\right\|}{\max \left(\left\|z_{\text {measured }}\right\|\right)}
$$

where $z_{o b s}$ is the variable calculated by the observer, $z_{\text {measured }}$ is the measured variable and $\max \left(\left\|z_{\text {measured }}\right\|\right)$ is the absolute maximum value of the measured variable during the test maneuver.

Figures 6 and 7 show lateral forces on the front and rear wheels, while figure 8 shows the sideslip angle evolution during the trajectory. These figures show that the observers are relatively good with respect to measurements. Some small differences during the trajectory are to be noted. These might be explained by neglected geometrical parameters, especially the cambers angles, which also produce a lateral forces component [22] and [23].

Comparing the two observers, we can see that $O_{U K F}$ is more efficient. In fact, during the time interval [12s-18s], when the vehicle is highly sollicitated, the observer $O_{E K F}$ does not converge well. This phenomenon is due to the intense nonlinearities of the vehicle dynamic equations. Therefore, the first order linearization of the EKF algorithm is not sufficient, and the errors of linearization are too important. The UKF algorithm shows his ability to overcome this difficulty. Consequently, we can deduce that the observer $O_{U K F}$ is the more appropriate estimator in our application. Table I presents maximum absolute values, normalized mean errors and normalized std for lateral tire forces and sideslip angle at the cog. Despite the simplicity of our chosen model, we can deduce that for this test, the performance of the observers, notably the $O_{U K F}$ is satisfactory, with normalized error globaly less than $8 \%$.

Given the vertical and lateral tire forces at each tire-road contact level, the estimation process is able to evaluate the used lateral friction coefficient $\mu$. This is defined as a ratio of friction force to normal force and it is given by:

$$
\mu_{i j}=\frac{F y_{i j}}{F z_{i j}}
$$

From figure 9, which shows the used lateral friction coefficients, we remark that the estimated $\mu_{i j}$ is close to the measured one. A closer investigation reveals that high $\mu_{i j}$ is detected during the trajectory, especially when the lateral acceleration is up to 0.6. During this maneuvre, we deduce
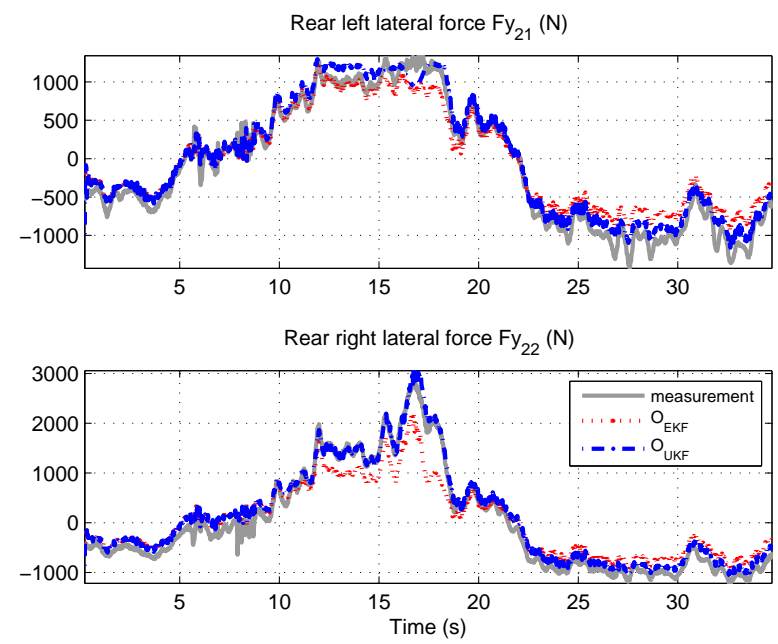

Fig. 7. Estimation of rear lateral tire forces.

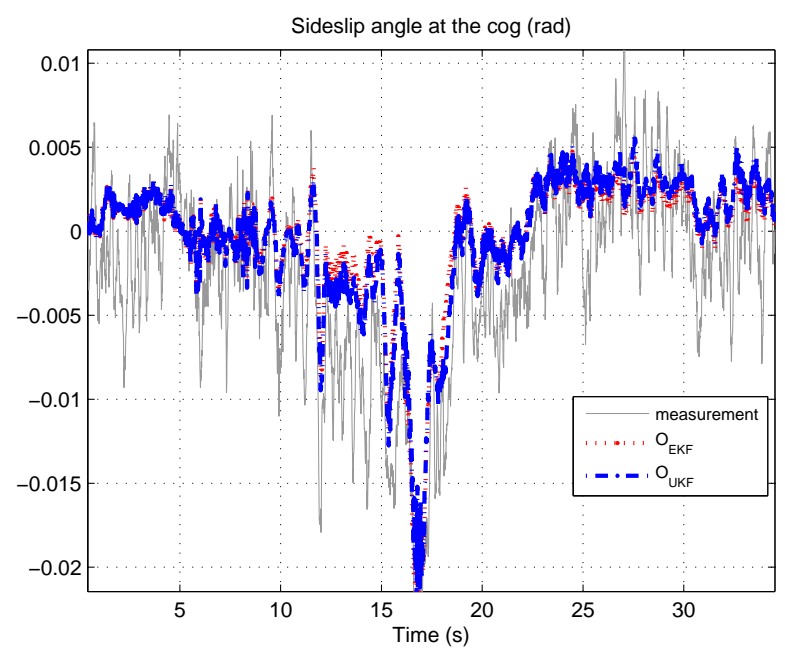

Fig. 8. Estimation of the sideslip angle at the cog.
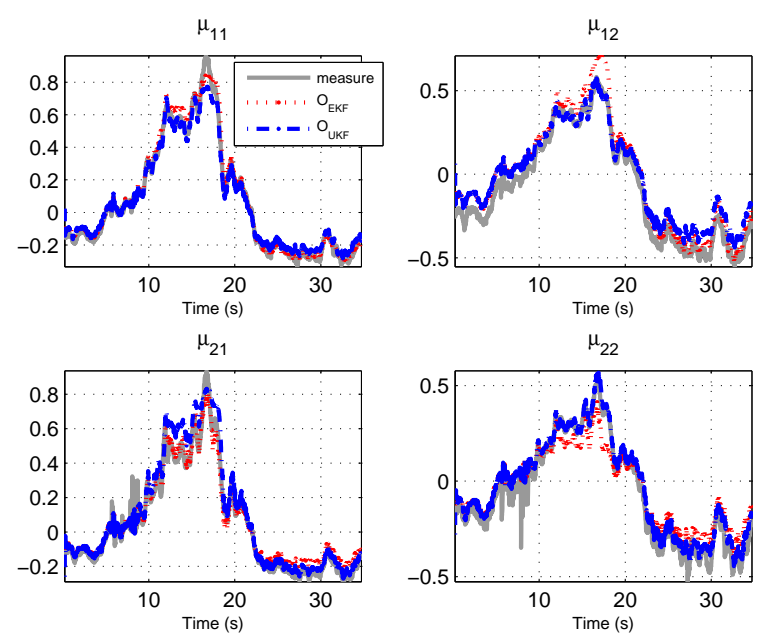

Fig. 9. Lateral friction coefficient 


\begin{tabular}{|c|c|cc|cr|}
\hline EKF UKF & Max \|\| & \multicolumn{2}{|c|}{ Mean $\%$} & \multicolumn{2}{|c|}{ Std \% } \\
\hline$F y_{11}$ & $2180(\mathrm{~N})$ & 4.87 & 5.02 & 3.75 & 3.51 \\
\hline$F y_{12}$ & $3617(\mathrm{~N})$ & 9.7 & 9.32 & 4.07 & 4.30 \\
\hline$F y_{21}$ & $1342(\mathrm{~N})$ & 11.98 & 7.55 & 9.14 & 5.33 \\
\hline$F y_{22}$ & $2817(\mathrm{~N})$ & 10.12 & 5.07 & 6.96 & 2.92 \\
\hline$\beta$ & 0.023 & 13.4 & 10.20 & 10.42 & 9.52 \\
\hline
\end{tabular}

TABLE I

OBSERVERS $O_{E K F}$ AND $O_{U K F}$ : MAXIMUM ABSOlute VAlUES, NORMALIZED MEAN ERRORS AND NORMALIZED STD.

that $\mu_{11}$ and $\mu_{21}$, which correspond to the non compressed tires (outside part of the vehicle during cornering), attained the limit for the dry road friction coefficient. In fact, dry road surfaces show a high friction coefficient in the range 0.9-1.1 (that results in safe driving on such surfaces), meaning that for this test the limits of handling were reached.

The friction coefficient evaluation is important for evaluating the ratio of the used friction and for determining the available remainder.

\section{CONCLUSION}

This paper has presented a new method for estimating lateral tire forces and sideslip angle, that is to say two of the most important parameters affecting vehicle stability and the risk of leaving the road. The two developed observers are derived from a simplified four-wheel vehicle model and are based respectively on extended and unscented Kalman filtering techniques. Tire-road interaction is represented by the Dugoff model. We then use the lateral friction model to evaluate the friction coefficient according to the estimated lateral and vertical forces from the whole estimation process. A comparison with real experimental data demonstrates the potential of the estimation process, showing that it may be possible to replace expensive correvit and dynamometric hub sensors by software observers that can work in real-time while the vehicle is in motion. This is one of the important results of our work. Another important result concerns the estimation of individual lateral forces acting on each tire of the vehicle, that is an evolution with respect to the current literature concerning the vehicle dynamic community.

Future studies will improve vehicle/road model in order to widen validity domains for the observer. Subsequent, vehicle/road models will take into account roll and vertical dynamics. Moreover, we note that the mean of the rear wheel speeds could be a poor approximation of the vehicle velocity in many situations (longitudinal tire slips, low road friction, ...). This shortcoming of the current design will be addressed in the future.

\section{REFERENCES}

[1] Y. Hsu and J. Chistian Gerdes, Experimental studies of using steering torque under various road conditions for sideslip and friction estimation, Proceedings of the 2007 IFAC Symposium on Advances in Automotive Control, Monterey, California.

[2] T. A. Wenzel, K. J. Burnham, M. V. Blundell and R. A. Williams, Estimation of the nonlinear suspension tyre cornering forces from experimental road test data, Vehicle System Dynamics, volume 44, pages 153-171, 2006.

[3] J. Dakhlallah, S. Glaser, S. Mammar and Y. SebsadjiTire-road forces estimation using extended Kalman filter and sideslip angle evaluation, American Control Conference, Seattle, Washington, U.S.A, June 2008.

[4] L. R. Ray, Nonlinear tire force estimation and road friction identifcation: simulation and experiments, Automatica, volume 33, no. 10, pages 1819-1833, 1997.
[5] G. Baffet, A. Charara and J. Stephant, Sideslip angle, lateral tire force and road friction estimation in simulations and experiments, IEEE International Conference on Control Applications, Munich, Germany, 2006.

[6] G. Baffet A. Charara, D. Lechner and D. Thomas, Experimental evaluation of observers for tire-road forces, sideslip angle and wheel cornering stiffness, Vehicle System Dynamics, volume 45, pages 191216 , june 2008 .

[7] M. A. Wilkin, W. J. Manning, D. A. Crolla, M. C. Levesley Use of an extended Kalman filter as a robust tyre force estimator, Vehicle System Dynamics, volume 44, pages 50-59, 2006.

[8] J. Stéphant, A. Charara and D. Meizel, Evaluation of sliding mode observer for vehicle sideslip angle, Control Engineering Practice. vol.15, pp. 803-812, July 2007.

[9] J. Stéphant, A. Charara and D. Meizel, Virtual sensor, application to vehicle sideslip angle and transversal forces, IEEE Transactions on Industrial Electronics. vol.51, no. 2, April 2004.

[10] M. Doumiati, G. Baffet, D. Lechner, A. Victorino and A. Charara, Embedded estimation of the tire/road forces and validation in a laboratory car, 9th International symposium on advanced vehicle control, Kobe, Japan, Octobre 2008.

[11] M. Doumiati, A. Victorino, A. Charara, D. Lechner and G. Baffet, An estimation process for vehicle wheel-ground contact normal forces, IFAC WC'08, Seoul Korea, july 2008.

[12] U. Kiencke and L. Nielsen, Automotive control systems, Springer, 2000.

[13] J. Dugoff, P. Fanches and L. Segel, An analysis of tire properties and their influence on vehicle dynamic performance, SAE paper (700377), 1970.

[14] Y. Hsu and J. Chistian Gerdes, Stabilization of a steer-by-wire vehicle at the limits of handling using feedback linearization, Procedings of IMECE2005, AMSE International Mechanical Engineering Congress and Exposition, Florida, U.S.A, 2005.

[15] P. Bolzern, F. Cheli, G. Falciola and F. Resta, Estimation of the nonlinear suspension tyre cornering forces from experimental road test data, Vehicle System Dynamics, volume 31, pages 23-24, 1999.

[16] H. Nijmeijer and A.J. Van der Schaft, Nonlinear dynamical control systems, Springer Verlag, 1991.

[17] G. Baffet, Développement et validation exprimentale d'observateurs des forces de contact pneumatique/chaussée d'une automobile, $\mathrm{Ph} . \mathrm{D}$ thesis, University of Technology of Compiègne, France, 2007.

[18] R.E. Kalman, A new approach to linear filtering and prediction problems, Transactions of the ASME- Journal of Basic Engineering. vol. 82. series D. pp.35-45,1960.

[19] G. Welch and G. Bishop, An introduction to the Kalman Filter, Course 8, University of North Carolina, chapel Hill, Departement of computer science, 2001.

[20] S. J. Julier and J. K. Uhlman, A new extension of the Kalman filter to nonlinear systems, International Symposium Aerospace/Defense Sensing, Simulation and Controls, Orlando, USA, 1997.

[21] S. J. Julier, J. K. Uhlman and H. F. Durrant-Whyte A new extension approach for filtering nonlinear systems, International Proceedings of the American Control Conference, Seattle, Washington, 1995.

[22] N. D. Smith, Understanding parameters influencing tire modeling, Colorado State University, Formula SAE Platform, 2004.

[23] W.F. Milliken and D.L. Milliken, Race car vehicle dynamics, Society of Automotive Engineers, Inc, U.S.A, 1995. 